\title{
Synergistic Effects of Branched-chain Amino Acids and Phenylalanine Addition on Major Volatile Compounds in Wine during Alcoholic Fermentation
}

\author{
Y.-Q. Wang ${ }^{1}$, D.-Q. Ye ${ }^{1,2}$, P.-T. Liu ${ }^{1}$, L.-L. Duan ${ }^{1}$, C.-Q. Duan ${ }^{1}$, G.-L. Yan ${ }^{1, *}$ \\ (1) Centre for Viticulture and Oenology, College of Food Science and Nutritional Engineering, China Agricultural University, \\ Beijing 100083, China \\ (2) College of Oenology, Northwest A\&F University, Yangling 712100, Shaanxi, China
}

Submitted for publication: June 2016

Accepted for publication: September 2016

Key words: wine fermentation, Saccharomyces cerevisiae, branched-chain amino acids, phenylalanine, aroma compounds

\begin{abstract}
The effects of adding branched-chain amino acids (BCAAs, including L-valine, L-leucine and L-isoleucine), L-phenylalanine and a mixture of them (BCAAs + Phe) on the fermentation profiles of wine yeast EC1118 and the production of volatile compounds were investigated in synthetic grape juice. The addition of selected amino acids had no considerable influence on the yeast growth and primary metabolites of the sugars. Adding BCAAs increased the production of higher alcohols, medium-chain fatty acids (MCFAs) and their corresponding ethyl esters. In comparison, adding Phe promoted the production of 2-phenylethanol, 2-phenylethyl acetate and ethyl esters of MCFAs. Nevertheless, the supplementation of BCAAs + Phe further heightened the production of MCFAs, acetate esters and ethyl esters of MCFAs compared to the single additions, but it attenuated the production of various higher alcohols (1-propanol, 2,3-butanediol and methionol) compared to the addition of BCAAs, and of 2-phenylethanol and 2-phenylethyl acetate contents compared to the Phe addition. These results suggest that adding BCAAs or Phe is an efficient way to adjust wine's aromatic composition and complexity. Meanwhile, the combined addition of BCAAs + Phe could be a potential tool to further manipulate wine's aromatic profile by accentuating or suppressing the formation of certain aroma compounds.
\end{abstract}

\section{INTRODUCTION}

The importance of yeast assimilable nitrogen (YAN) to yeast growth and fermentation metabolism is well known in the wine industry. The composition and amount of YAN can regulate the formation of yeast biomass and the fermentation rate (Bell \& Henschke, 2005). Moreover, the nitrogen status of must can affect the production by Saccharomyces cerevisiae of many volatile compounds that contribute to wine flavour, including higher alcohols, short- to medium-chain fatty acids (MCFAs), and their ethyl ester or acetate ester derivatives (Swiegers et al., 2005). The principal sources of YAN in grape must are $\alpha$-amino acids and ammonium. Insufficient nitrogen can lead to a delay in or even a cessation of fermentation. Thus, ammonium salts are usually added to grape juice to prevent these problems in wineries. However, the addition of large quantities of ammonium can result in microbiological instability and the increased production of ethyl acetate, acetic acid and carcinogenic ethyl carbamate (Vilanova et al., 2007; Garde-Cerdán \& Ancín-Azpilicueta, 2008; Torrea et al., 2011).
Supplying amino acids is an alternative approach to accelerate cell growth and alter the aroma profile of the wine produced (Hernández-Orte et al., 2006; Garde-Cerdán \& Ancín-Azpilicueta, 2008). Generally, the formation of higher alcohols and their corresponding acetate esters is directly proportional to the quantity of amino acids added to the juice (Trinh et al., 2010; Lee et al., 2013). As reported by Chen et al. (2014), supplementation of lychee juice with Phe resulted in significantly higher amounts of 2-phenylethyl alcohol, 2-phenylethyl acetate, 2-phenylethyl isobutyrate and 2-phenylethyl hexanoate. More 2-phenylethyl alcohol was obtained when adding Asp, Phe, Thr and Ala to Merlot juice (Hernández-Orte et al., 2006). However, inconsistent results have also been reported. Hernández-Orte et al. (2005) found that the simultaneous doubling of the level of amino acids in Airen must reduced the content of 2-phenylethanol, methionol and isoamyl alcohol in the wine and increased the contents of propanoic acid and isobutanol. These discrepancies were ascribed to the different compositions

*Corresponding author: E-mail address: glyan@cau.edu.cn [Tel: +86-10-62737039; Fax: +86-10-62738658]

Acknowledgements: This work was financially supported by the China Agriculture Research System (CARS-30) and the Chinese Universities Scientific Fund (2016SP002) 
of the amino acids (Procopio et al., 2015). It is well known that not all nitrogen compounds that exist in grape must support yeast growth equally well. Yeast preferentially uses substrates (glutamine and ammonium) that allow the best growth, through a regulation mechanism called nitrogen catabolite repression (NCR) (Crépin et al., 2012). Thus, the variation in amino acid profiles can influence the order in which different amino acids are used by yeast, which in turn affects the ratio of secondary metabolites produced (Hernández-Orte et al., 2002).

Branched-chain amino acids (BCAAs, including L-valine, L-leucine and L-isoleucine) and L-phenylalanine are very important flavour precursors in grape must. These amino acids can be assimilated by yeast via the Ehrlich pathway to produce higher alcohols during fermentation (Hazelwood et al., 2008). These alcohols, together with acids, can form esters such as isoamyl acetate and phenylethyl acetate, which contribute distinctly to the pleasant fruity aroma of wine. Considering the importance of BCAAs and Phe in the formation of fermented flavour compounds, this study was directed at investigating the influence of the combined addition of BCAAs and Phe on the production of yeast-derived volatiles. To facilitate this investigation, a simplified, chemically defined medium (MS300) that resembles the nutrient composition of grape juice was used.

\section{MATERIALS AND METHODS}

\section{Strain and culture medium}

Fermentations were carried out with the commercial Saccharomyces cerevisiae var. bayanus strain EC1118 (Lallemand Inc., Blagnac, France). This commercial wine yeast, used worldwide for both red and white winemaking, is considered a fast and robust fermenting strain, with a neutral contribution to the wine aroma (Molina et al., 2007). The chemically defined medium MS300, similar in composition to typical grape juice (Bely et al., 1990), was used with a little modification. It contained the following ingredients: $240 \mathrm{~g} / \mathrm{L}$ of glucose; $6 \mathrm{~g} / \mathrm{L}$ of citric acid and 6 $\mathrm{g} / \mathrm{L}$ of malic acid; mineral salts (in $\mathrm{mg} / \mathrm{L}$ ): $\mathrm{KH}_{2} \mathrm{PO}_{4}(750)$, $\mathrm{K}_{2} \mathrm{SO}_{4}(500), \quad \mathrm{MgSO}_{4} \cdot 7 \mathrm{H}_{2} \mathrm{O}$ (250), $\mathrm{CaCl}_{2} \cdot 2 \mathrm{H}_{2} \mathrm{O}$ (155), $\mathrm{NaCl}(200), \mathrm{MnSO}_{4} \cdot \mathrm{H}_{2} \mathrm{O}(4), \mathrm{ZnSO}_{4}(4), \mathrm{CuSO}_{4} \cdot 5 \mathrm{H}_{2} \mathrm{O}(1)$, $\mathrm{KI}(1), \mathrm{CoCl}_{2} \cdot 6 \mathrm{H}_{2} \mathrm{O}(0.4), \mathrm{H}_{3} \mathrm{BO}_{3}(1), \mathrm{NaMoO}_{4} \cdot 2 \mathrm{H}_{2} \mathrm{O}$ (1); vitamins (in $\mathrm{mg} / \mathrm{L}$ ): myoinositol (20), nicotinic acid (2), calcium pantothenate (1.5), thiamine hydrochloride $(0.25)$, pyridoxine hydrochloride $(0.25)$, biotin $(0.003)$; anaerobic growth factors: $15 \mathrm{mg} / \mathrm{L}$ ergosterol, $0.5 \mathrm{~mL} / \mathrm{L}$ of Tween 80 ; and nitrogen source (in mg/L): ammonium chloride (460), L-proline (612.6), L-glutamine (505.3), L-arginine (374.4), L-tryptophan (179.3), L-alanine (145.3), L-glutamic acid
(120.4), L-serine (78.5), L-threonine (75.9), L-aspartic acid (44.5), L-leucine (48.4), L-isoleucine (32.7), L-valine (44.5), L-phenylalanine (37.9), L-histidine (32.7), L-methionine (31.4), L-tyrosine (18.3), L-glycine (18.3), L-lysine (17.0) and L-cysteine (13.1). The ammonium salt and $\alpha$-amino acids (except proline) in the medium were considered yeast assimilable nitrogen. The medium $\mathrm{pH}$ was adjusted to 3.3. Based on MS300, BCAAs and/or Phe were added to the medium. The amino acid contents added were in the normal concentration range for these amino acids in grape must (Bell \& Henschke, 2005). The nitrogen conditions of the treatments are listed in Table 1.

\section{Fermentation conditions}

The inoculum for all experiments was prepared by pregrowing the yeast in $500 \mathrm{~mL}$ shake flasks containing $300 \mathrm{~mL}$ of the YPD medium $(20 \mathrm{~g} / \mathrm{L}$ of glucose, $10 \mathrm{~g} / \mathrm{L}$ of peptone and $5 \mathrm{~g} / \mathrm{L}$ of yeast extract purchased from Oxoid) at $30^{\circ} \mathrm{C}$ with shaking $(150 \mathrm{rpm})$ overnight. This starter culture was used to inoculate the experiment cultures with an initial viable population of $10^{6} \mathrm{CFU} / \mathrm{mL}$. Cells were harvested by centrifuging and were washed twice with sterile water. The fermentations were carried out in $500 \mathrm{~mL}$ flasks filled with $350 \mathrm{~mL}$ of the chemically defined medium and fitted with a fermentation lock and a puncture needle for sampling. All fermentations were maintained at $25^{\circ} \mathrm{C}$ without shaking. All fermentations were performed in triplicate. The progress of fermentation was monitored by periodic measurement of cell density (optical density at $660 \mathrm{~nm}, \mathrm{OD}_{600}$ ) and sugar consumption. Samples were taken and centrifuged at $5000 \mathrm{rpm}$ for $10 \mathrm{~min}$ at $4^{\circ} \mathrm{C}$, and the cell-free supernatants were stored at $-20^{\circ} \mathrm{C}$ before analysis.

\section{Analytical methods}

In all fermentation samples, the concentrations of glucose, fructose, ethanol, glycerol, acetic acid, malic acid, citric acid, lactic acid and succinic acid were determined using the HPLC method described by Verwaal et al. (2010). The system was equipped with a HPX-87H Aminex ion-exchange column (300 mm $\times 7.8 \mathrm{~mm}$, Bio-Rad Laboratories, Hercules, CA, USA), with $5 \mathrm{mM}$ sulfuric acid as the mobile phase. Glucose, fructose, ethanol and glycerol were detected with a refractive index detector, and the column was maintained at $45^{\circ} \mathrm{C}$. All of the acids were detected with a photodiode array detector at $214 \mathrm{~nm}$, and the column was maintained at $60^{\circ} \mathrm{C}$. Samples were diluted and filtered through $0.22 \mu \mathrm{m}$ membrane filters (purchased from DIKMA) prior to the HPLC analysis.

Amino acids and ammonium in the medium were analysed using the HPLC method developed previously

TABLE 1

Concentration of amino acids and yeast assimilable nitrogen in different treatments.

\begin{tabular}{|c|c|c|c|c|c|c|}
\hline No. & Treatments & $\begin{array}{l}\text { Val } \\
(\mathrm{mg} / \mathrm{L})\end{array}$ & $\begin{array}{l}\text { Leu } \\
(\mathrm{mg} / \mathrm{L})\end{array}$ & $\begin{array}{l}\text { Ile } \\
(\mathrm{mg} / \mathrm{L})\end{array}$ & $\begin{array}{l}\text { Phe } \\
(\mathrm{mg} / \mathrm{L})\end{array}$ & $\begin{array}{l}\text { YAN } \\
(\mathrm{mg} \mathrm{N} / \mathrm{L})\end{array}$ \\
\hline 1 & Control & 44.5 & 48.4 & 32.7 & 37.9 & 300 \\
\hline 2 & BCAAs & 133.5 & 145.2 & 98.1 & 37.9 & 329 \\
\hline 3 & Phe & 44.5 & 48.4 & 32.7 & 113.7 & 307 \\
\hline 4 & BCAAs + Phe & 133.5 & 145.2 & 98.1 & 113.7 & 335 \\
\hline
\end{tabular}


in our laboratory (Wang et al., 2014). Samples were precolumn derivatised with diethyl ethoxymethylenemalonate (DEEMM). The HPLC analysis was performed using an Agilent 1200 series instrument. Chromatography separation was performed on an Agilent ZORBAX SB-C18 Rapid Resolution HT column ( $3.0 \mathrm{~mm} \times 50 \mathrm{~mm}, 1.8$ micron). For detection, a photodiode array detector monitoring at $280 \mathrm{~nm}$ was used.

Volatile compounds in the wines were analysed using the HS-SPME-GC-MS method developed by Zhang et al. (2011). Samples were extracted with 50/30 $\mu \mathrm{m}$ DVB/CAR/ PDMS fibre (Supelco, Bellefonte, PA, USA). The GC-MS system used was an Agilent 7890A GC equipped with an Agilent $5975 \mathrm{C}$ mass spectrometer. The column used was a $60 \mathrm{~m} \times 0.25 \mathrm{~mm}$ HP-INNOWAX capillary with $0.25 \mu \mathrm{m}$ film thickness (J\&W Scientific, Folsom, CA, USA). The carrier gas was helium at a flow rate of $1.0 \mathrm{~mL} / \mathrm{min}$. The oven's starting temperature was $50^{\circ} \mathrm{C}$, which was held for $1 \mathrm{~min}$, then raised to $220^{\circ} \mathrm{C}$ at a rate of $3^{\circ} \mathrm{C} / \mathrm{min}$ and held for 5 min. Electron ionisation (electron impact mode at $70 \mathrm{eV}$ ) mass spectrometric data from m/z 30 to 350 was collected. All determinations were performed in triplicate. The detailed identification and quantification information is listed in Supplementary Table 1.

\section{Statistical analysis}

Mean and standard deviation values were calculated on the basis of the data obtained from the triplicate fermentations performed for each treatment. Significant differences in metabolites among treatments were determined using the one-way analysis of variance (ANOVA) test, employing Duncan's multiple range test at a significance level of $p<0.05$ (SPSS 17.0 statistical software, SPSS Inc., Chicago, IL, USA).

\section{RESULTS AND DISCUSSION}

\section{Yeast growth and fermentation profiles}

As shown in Fig. 1, the supplementation of BCAAs or Phe to the medium had little effect on the fermentation kinetics, except for the combined addition of BCAAs + Phe, which slightly stimulated cell growth and the sugar consumption rate, especially for the second half of the fermentation (residual sugar less than 50\%). According to previous results, once the YAN is sufficient for yeast growth (above $140 \mathrm{mg}$ $\mathrm{N} / \mathrm{L}$ ), there is little variation in the rate of fermentation in response to nitrogen supplementation (Hernández-Orte et al., 2005; Garde-Cerdán \& Ancín-Azpilicueta, 2008).

Ethanol and glycerol production were not significantly affected by amino acid addition compared to the control (Table 2). Their concentrations ranged from $11.31 \%$ to $11.85 \%(\mathrm{~V} / \mathrm{V})$ and $5.28 \mathrm{~g} / \mathrm{L}$ to $5.48 \mathrm{~g} / \mathrm{L}$ respectively. The $\mathrm{pH}$ value was decreased from 3.27 in the control wine to approximately 3.05 by amino acid addition. Except for acetic acid and succinic acid, the contents of malic acid, citric acid and lactic acid showed little variation with amino acid additions. The level of malic acid in the wines was nearly

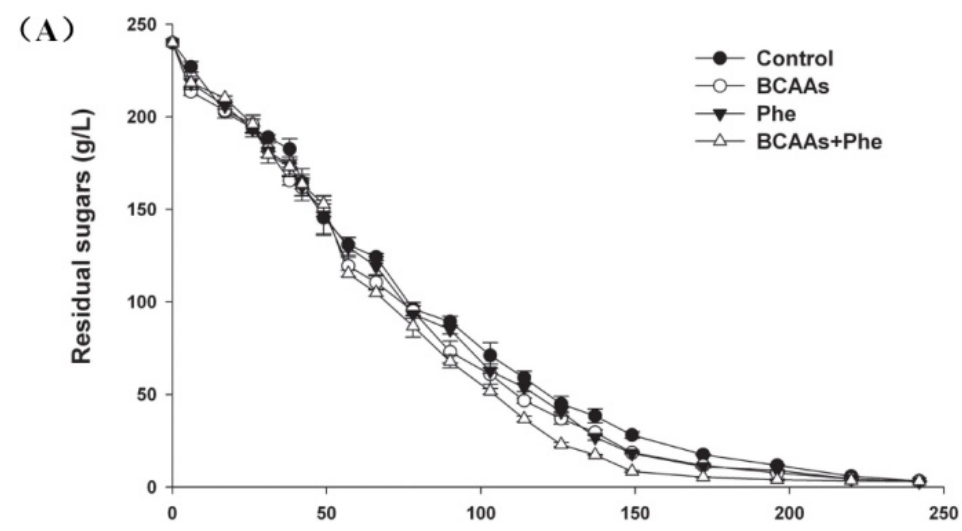

(B)

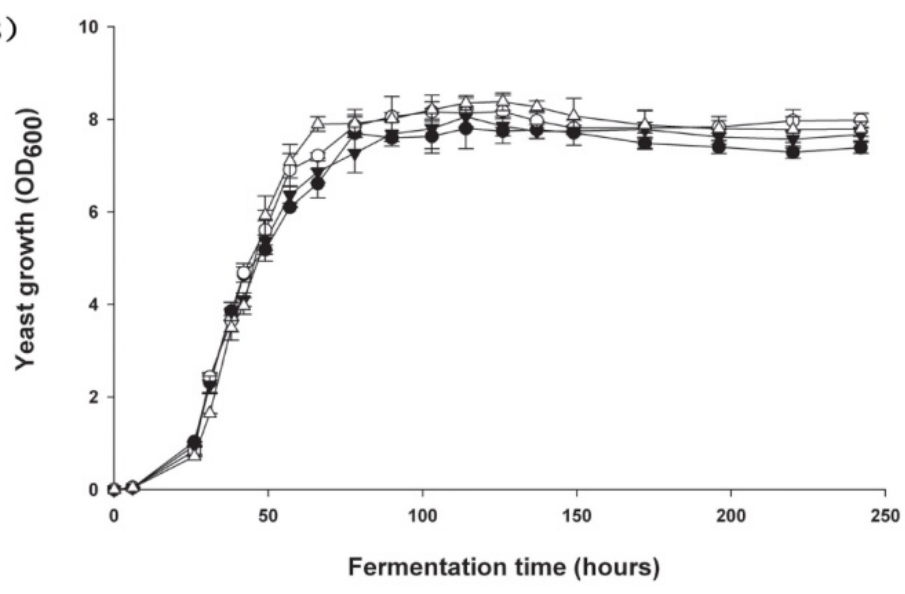

FIGURE 1

Sugar consumption (A) and yeast growth (B) during the fermentations with the addition of BCAAs and Phe. 
TABLE 2

General oenological parameters of wines obtained from the fermentation of synthetic grape juice with different initial concentrations of BCAAs and Phe. Values (mean $\pm \mathrm{SD}, \mathrm{n}=3$ ) of the same parameter (compound) followed by the same lowercase letters indicate no significant difference $(p<0.05)$.

\begin{tabular}{lllll}
\hline Treatments & Control & BCAAs & Phe & BCAAs + Phe \\
\hline Alcohol (V/V, \%) & $11.85 \pm 0.12 \mathrm{a}$ & $11.48 \pm 0.43 \mathrm{a}$ & $11.31 \pm 0.38 \mathrm{a}$ & $11.73 \pm 0.23 \mathrm{a}$ \\
Glycerol (g/L) & $5.35 \pm 0.19 \mathrm{a}$ & $5.28 \pm 0.19 \mathrm{a}$ & $5.48 \pm 0.32 \mathrm{a}$ & $5.46 \pm 0.12 \mathrm{a}$ \\
$\mathrm{pH}$ & $3.27 \pm 0.02 \mathrm{a}$ & $3.07 \pm 0.01 \mathrm{~b}$ & $3.05 \pm 0.01 \mathrm{~b}$ & $3.06 \pm 0.04 \mathrm{~b}$ \\
Residual YAN (mg N/L) & $37.17 \pm 0.62 \mathrm{c}$ & $39.68 \pm 1.31 \mathrm{~b}$ & $37.18 \pm 1.23 \mathrm{c}$ & $43.14 \pm 1.73 \mathrm{a}$ \\
Acetic acid (g/L) & $0.77 \pm 0.05 \mathrm{~b}$ & $0.79 \pm 0.01 \mathrm{ab}$ & $0.83 \pm 0.02 \mathrm{a}$ & $0.79 \pm 0.01 \mathrm{ab}$ \\
Malic acid (g/L) & $5.73 \pm 0.40 \mathrm{a}$ & $5.48 \pm 0.02 \mathrm{a}$ & $5.57 \pm 0.21 \mathrm{a}$ & $5.55 \pm 0.14 \mathrm{a}$ \\
Citric acid (g/L) & $4.44 \pm 0.25 \mathrm{a}$ & $4.35 \pm 0.10 \mathrm{a}$ & $4.41 \pm 0.15 \mathrm{a}$ & $4.48 \pm 0.15 \mathrm{a}$ \\
Lactic acid (g/L) & $0.23 \pm 0.01 \mathrm{a}$ & $0.23 \pm 0.01 \mathrm{a}$ & $0.26 \pm 0.02 \mathrm{a}$ & $0.26 \pm 0.01 \mathrm{a}$ \\
Succinic acid (g/L) & $0.36 \pm 0.03 \mathrm{a}$ & $0.30 \pm 0.00 \mathrm{bc}$ & $0.34 \pm 0.02 \mathrm{ab}$ & $0.29 \pm 0.01 \mathrm{c}$ \\
\hline
\end{tabular}

the same as in the initial medium, confirming that the strain EC1118 could not efficiently degrade malic acid because of the poor uptake of malic acid by simple diffusion and a very low substrate affinity of malic enzyme, which could be dysfunctional under winemaking conditions (Redzepovic et al., 2003; Chen et al., 2014; Su et al., 2014). Adding BCAAs and BCAAs + Phe led to a slight but significant decrease in the succinic acid contents compared to the control and Pheadded fermentation. This was consistent with the results of Torrea et al. (2011), who reported a decreased succinate concentration with the addition of inorganic and organic nitrogen. These results collectively suggest that adding BCAAs and/or Phe to the medium containing sufficient YAN did not have a considerable influence on the cell growth and primary metabolites of sugar.

We also determined the consumption profiles of amino acids in different treatments. The consumption of specific amino acids was determined by calculating the ratio of consumed amino acid content to the initial content in the medium (Supplementary Table 2). The addition of single BCAAs decreased the consumption ratios of Val, Phe, Tyr and Cys compared to the control, especially Tyr, as its consumption ratio dropped from $66.9 \%$ to $39.7 \%$. In comparison, Phe addition only caused a decreased utilisation of Glu and Cys. The consumption of more types of amino acids was inhibited in the combined addition of BCAAs + Phe medium, including Asp, Val, Phe, Cys and Tyr. Unlike other amino acids, Ser consumption increased with the combined addition of BCAAs + Phe medium $(80.2 \%)$ compared to the control $(69.8 \%)$

\section{Volatile compounds in the wines supplemented with ami- no acids}

After alcoholic fermentation, the concentrations of the most important aroma-active compounds were analysed (Table 3). The addition of BCAAs and/or Phe significantly affected the synthesis of wine aroma compounds. Generally, the total production of volatile aroma compounds was promoted by the addition of selected amino acids.

\section{Higher alcohols}

Higher alcohols were the main abundant group of volatiles in all wines. A positive relationship between higher alcohol production and amino acid supplementation was found. BCAA addition resulted in a $34.4 \%$ increase in 3-methyl butanol compared to the control. This was reasonable, considering the mechanism involved in the Ehrlich pathway. BCAA addition also increased the production of 2-phenylethanol, 1-propanol and 2,3-butanediol. As a result, the total production of higher alcohols was $81.6 \%$ higher than that of the control.

The highest increase in higher alcohol was observed in 2-phenylethanol with Phe-added fermentation. Its concentration increased by $157.4 \%$ and $80.6 \%$ compared to the control and the BCAA-added wines respectively. This result was consistent with the data of Chen et al. (2014), who found that supplementation of lychee juice with Phe significantly promoted the formation of 2-phenylethyl alcohol. Unlike BCAA addition, Phe addition had no obvious effect on the production of other higher alcohols.

Although they still presented a promotion effect compared to the control, most higher alcohols showed a lesser extent of promotion in wines to which BCAAs + Phe had been added compared to single additions, except for 2-methyl propanol. This suggests that the combined addition of BCAAs + Phe could weaken the promotion effect of higher alcohol production induced by the single addition of the amino acid(s). Moreira et al. (2011) also observed that branched-chain alcohols, including isobutyl alcohol and isoamyl alcohol, were decreased in wine supplemented with DAP, which could be explained by the interaction of different nitrogen sources because ammonium is preferentially taken up and consumed by yeast cells compared to BCAAs (Crépin et al., 2012).

Higher alcohols could contribute a masking effect of fruity aromas in wines, and they are able to communicate alcoholic and solvent notes to the wine only in certain aromatic contexts (Cameleyre et al., 2015; De-la-FuenteBlanco et al., 2016). Compared to Phe, increasing the initial BCAA concentration in the medium could result in the higher formation of higher alcohols. As a result, controlling 


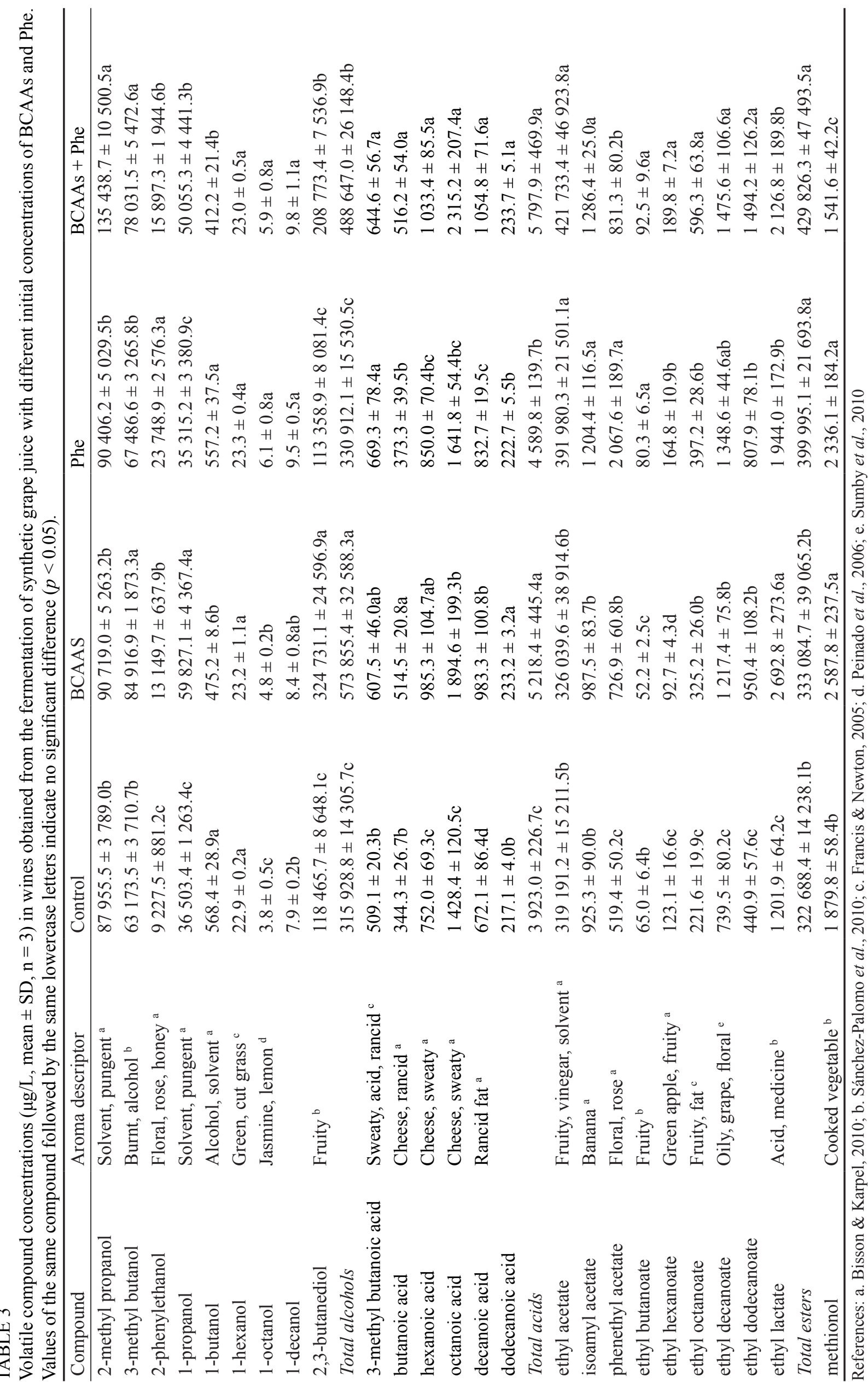


the BCAA level in grape must is a potential way to decrease the adverse effect of higher alcohols on wine fruity aromas.

Acids

The formation of total volatile acids (except acetic acid) was proportional to the quantity of amino acids added to the medium. 3-Methyl butanoic acid was the only branched-chain acid quantified in this study, and it was derived from Leu metabolism. The addition of selected amino acids promoted its biosynthesis compared to the control. Medium-chain fatty acids (C6 to $\mathrm{C} 12$ ) are another significant group of volatiles that can directly contribute to the flavour of wine or serve as substrates that participate in the formation of ethyl esters. A positive relationship was observed between the production of MCFAs and the addition of amino acid(s). BCAA addition showed higher promotion of MCFA production than Phe addition did (total content $4.10 \mathrm{mg} / \mathrm{L}$ vs. $3.55 \mathrm{mg} / \mathrm{L}$ ). This promotion effect became more pronounced in the mixed addition wines: $13.1 \%, 31.0 \%$ and $51.1 \%$ increases in the MCFA concentrations were observed in the combined additions of BCAAs + Phe compared to BCAA addition, Phe addition and the control respectively. These results suggest that BCAAs and Phe cooperatively promoted the production of MCFA. The synthesis of fatty acid could be improved by the addition of amino acids, which help yeast cells adapt their membrane fatty acid composition and resist the harsh environments during alcoholic fermentation (Torija et al., 2003). Therefore, the increased MCFAs from amino acid supplementation might be involved in the promotion of fatty acid synthesis.

Fatty acids play an important role in the complexity of wine aroma, and they could contribute a fresh flavour to wine; however, high contents can generate cheesy, sweaty or rancid odours in wine (Table 3). In the present study, the total fatty acid concentration in the wines was below the concentration that could impair wine aroma (above $20 \mathrm{mg} / \mathrm{L}$ ) (Pozo-Bayón et al., 2005).

\section{Esters}

Acetate esters are important aroma compounds and generate the desired fruity flavour in wine. They are produced by the reaction of acetyl-CoA with higher alcohols that are formed from the metabolism of amino acids or carbohydrates (Bisson \& Karpel, 2010). The content of acetate esters showed an increased trend in response to amino acid(s) supplementation, but varied with the type of amino acid added. Surprisingly, BCAA supplementation slightly improved isoamyl acetate production, but significantly increased 2-phenylethyl acetate concentration ( $40.1 \%$ higher than the control). In comparison, Phe addition resulted in $30.2 \%$ and $298.4 \%$ increases in isoamyl acetate and 2-phenylethyl acetate compared to the control. The combined addition caused a $39.0 \%$ increase in isoamyl acetate compared to the control, but only a $60.1 \%$ increase in 2-phenylethyl acetate was obtained relative to the control, which was much lower than that of the Phe addition. 2-Phenylethyl acetate is an important volatile compound in wine and is associated with honey, fruity and floral aromas. These results suggest that the combined addition of BCAAs + Phe was unfavourable for the synthesis of 2-phenylethyl acetate compared to single Phe addition, i.e. the presence of BCAAs could attenuate the increased magnitude of 2-phenylethyl acetate caused by Phe addition. The level of 2-phenylethyl acetate was directly proportional to the quantity of Phe added to the must, and thus the increase in 2-phenylethanol accumulation (Lee et al., 2013). As a result, the reduction of 2-phenylethyl acetate in the mixed addition wines could be due to the lower level of 2-phenylethanol produced.

Fatty acid ethyl esters are important flavour-active metabolites that are responsible for the highly desired fruit aroma characters in wine. They are generated by an alcohol and acyl group derived from activated MCFAs (Bisson \& Karpel, 2010). The addition of BCAAs and Phe stimulated or decreased the production of ethyl esters of MCFAs, depending on the type of amino acids added and esters. The addition of BCAAs promoted the production of ethyl octanoate, ethyl decanoate, ethyl dodecanoate and ethyl lactate, but decreased the content of ethyl butanoate and ethyl hexanoate. Phe addition enhanced all ethyl esters of MCFAs, and the total concentration was $69.9 \%$ higher than that of the control. The total content of ethyl esters was further heightened by the mixed addition of BCAAs + Phe, which obtained a 306.2\% increase compared to the control, suggesting that BCAAs + Phe had a synergic promotion effect on the biosynthesis of ethyl esters of MCFAs. Sufficient MCFAs are crucial for the biosynthesis of pleasant ethyl esters (Saerens et al., 2008); therefore, the increase in ethyl esters induced by amino acid addition could be ascribed to the accumulation of the substrate MCFAs in the medium.

\section{S-containing volatile compound}

Methionol, the main S-containing compound, was quantified in all the wines. It contributed a cooked vegetable aroma. There was a positive relationship between its production and the single addition of BCAAs or Phe. However, the combined addition of BCAAs + Phe eliminated this effect and even decreased its concentration below the control. The combined addition of BCAAs + Phe is a potential approach to decreasing the synthesis of methionol in wine.

\section{CONCLUSIONS}

This study found that the mixed addition of BCAAs + Phe had a more complicated influence on the production of volatile compounds compared to the single addition of BCAAs or Phe: it further heightened the production of MCFAs, acetate esters and ethyl esters of MCFAs; lowered the production of some higher alcohols (1-propanol, 2,3-butanediol and methionol) induced by BCAA addition; and lowered 2-phenylethanol and 2-phenylethyl acetate compared to Phe addition. These results suggest that adding BCAAs or Phe was an efficient tool to manipulate the aroma sensory quality of wines. However, the combined addition of BCAAs + Phe could result in a distinct influence on the production of volatile compounds by accentuating or suppressing the formation of certain aroma compounds, which might be a potential way to produce wines with divergent aroma attributes. 


\section{LITERATURE CITED}

Bell, S.-J. \& Henschke, P.A., 2005. Implications of nitrogen nutrition for grapes, fermentation and wine. Aust. J. Grape Wine Res. 11, 242-295.

Bely, M., Sablayrolles, J.-M. \& Barre, P., 1990. Automatic detection of assimilable nitrogen deficiencies during alcoholic fermentation in oenological conditions. J. Ferm. Bioeng. 70, 246-252.

Bisson, L.F. \& Karpel, J.E., 2010. Genetics of yeast impacting wine quality. Annu. Rev. Food Sci. Technol. 1, 139-162.

Cameleyre, M., Lytra, G., Tempere, S. \& Barbe, J.-C., 2015. Olfactory impact of higher alcohols on red wine fruity ester aroma expression in model solution. J. Agric. Food Chem. 63, 9777-9788.

Chen, D., Chia, J.Y. \& Liu, S.-Q., 2014. Impact of addition of aromatic amino acids on non-volatile and volatile compounds in lychee wine fermented with Saccharomyces cerevisiae MERIT.ferm. Int. J. Food Microbiol. 170, 12-20.

Crépin, L., Nidelet, T., Sanchez, I., Dequin, S. \& Camarasa, C., 2012. Sequential use of nitrogen compounds by Saccharomyces cerevisiae during wine fermentation: A model based on kinetic and regulation characteristics of nitrogen permeases. Appl. Environ. Microbiol. 78, 8102-8111.

De-la-Fuente-Blanco, A., Sáenz-Navajas, M.P. \& Ferreira, V., 2016. On the effects of higher alcohols on red wine aroma. Food Chem. 210, 107-114.

Francis, I.L. \& Newton, J.L., 2005. Determining wine aroma from compositional data. Aust. J. Grape Wine Res. 11, 114-126.

Garde-Cerdán, T. \& Ancín-Azpilicueta, C., 2008. Effect of the addition of different quantities of amino acids to nitrogen-deficient must on the formation of esters, alcohols, and acids during wine alcoholic fermentation. LWT-Food Sci. Technol. 41, 501-510.

Hazelwood, L.A., Daran, J.-M., Van Maris, A.J., Pronk, J.T. \& Dickinson, J.R., 2008. The Ehrlich pathway for fusel alcohol production: A century of research on Saccharomyces cerevisiae metabolism. Appl. Environ. Microbiol. 74, 2259-2266.

Hernández-Orte, P., Cacho, J.F. \& Ferreira, V., 2002. Relationship between varietal amino acid profile of grapes and wine aromatic composition. Experiments with model solutions and chemometric study. J. Agric. Food Chem. 50, 2891-2899.

Hernández-Orte, P., Ibarz, M.J., Cacho, J. \& Ferreira, V., 2005. Effect of the addition of ammonium and amino acids to musts of Airen variety on aromatic composition and sensory properties of the obtained wine. Food Chem. 89, 163-174.

Hernández-Orte, P., Ibarz, M.J., Cacho, J. \& Ferreira, V., 2006. Addition of amino acids to grape juice of the Merlot variety: Effect on amino acid uptake and aroma generation during alcoholic fermentation. Food Chem. 98, 300-310.

Lee, P.-R., Toh, M., Yu, B., Curran, P. \& Liu, S.-Q., 2013. Manipulation of volatile compound transformation in durian wine by nitrogen supplementation. Int. J. Food Sci. Tech. 48, 650-662.

Molina, A.M., Swiegers, J.H., Varela, C., Pretorius, I.S. \& Agosin, E., 2007. Influence of wine fermentation temperature on the synthesis of yeast-derived volatile aroma compounds. Appl. Microbiol. Biotechnol. 77, 675-687.

Moreira, N., De Pinho, P.G., Santos, C. \& Vasconcelos, I., 2011. Relationship between nitrogen content in grapes and volatiles, namely heavy sulphur compounds, in wines. Food Chem. 126, 1599-1607.

Peinado, R.A., Mauricio, J.C. \& Moreno, J., 2006. Aromatic series in sherry wines with gluconic acid subjected to different biological aging conditions by Saccharomyces cerevisiae var. capensis. Food Chem. 94, 232-239.
Pozo-Bayón, M., G-Alegría, E., Polo, M.C., Tenorio, C., Martín-Álvarez, P.J., Calvo de la Banda, M.T., Ruiz-Larrea, F. \& Moreno-Arribas, M.V., 2005. Wine volatile and amino acid composition after malolactic fermentation: Effect of Oenococcus oeni and Lactobacillus plantarum starter cultures. J. Agric. Food Chem. 53, 8729-8735.

Procopio, S., Sprung, P. \& Becker, T., 2015. Effect of amino acid supply on the transcription of flavour-related genes and aroma compound production during lager yeast fermentation. LWT-Food Sci. Technol. 63, 289-297.

Redzepovic, S., Orlic, S., Majdak, A., Kozina, B., Volschenk, H. \& ViljoenBloom, M., 2003. Differential malic acid degradation by selected strains of Saccharomyces during alcoholic fermentation. Int. J. Food Microbiol. 83, 49-61.

Saerens, S., Delvaux, F., Verstrepen, K., Van Dijck, P., Thevelein, J. \& Delvaux, F., 2008. Parameters affecting ethyl ester production by Saccharomyces cerevisiae during fermentation. Appl. Environ. Microbiol. 74, 454-461.

Sánchez-Palomo, E., Gómez García-Carpintero, E., Alonso-Villegas, R. \& González-Viñas, M.A., 2010. Characterization of aroma compounds of Verdejo white wines from the La Mancha region by odour activity values. Flavour Frag. J. 25, 456-462.

Su, J., Wang, T., Wang, Y., Li, Y.Y. \& Li, H., 2014. The use of lactic acidproducing, malic acid-producing, or malic acid-degrading yeast strains for acidity adjustment in the wine industry. Appl. Microbiol. Biotechnol. 98 , 2395-2413

Sumby, K.M., Grbin, P.R. \& Jiranek, V., 2010. Microbial modulation of aromatic esters in wine: Current knowledge and future prospects. Food Chem. 121, 1-16.

Swiegers, J.H., Bartowsky, E.J., Henschke, P.A. \& Pretorius, I.S., 2005. Yeast and bacterial modulation of wine aroma and flavour. Aust. J. Grape Wine Res. 11, 139-173.

Torija, M.J., Beltran, G., Novo, M., Poblet, M., Rozès, N., Guillamón, J.M. $\&$ Mas, A., 2003. Effect of the nitrogen source on the fatty acid composition of Saccharomyces cerevisiae. Food Microbiol. 20, 255-258.

Torrea, D., Varela, C., Ugliano, M., Ancin-Azpilicueta, C., Leigh Francis, I. \& Henschke, P.A., 2011. Comparison of inorganic and organic nitrogen supplementation of grape juice - Effect on volatile composition and aroma profile of a Chardonnay wine fermented with Saccharomyces cerevisiae yeast. Food Chem. 127, 1072-1083

Trinh, T.T.T., Woon, W.Y., Yu, B., Curran, P. \& Liu, S.-Q., 2010. Effect of L-isoleucine and L-phenylalanine addition on aroma compound formation during longan juice fermentation by a co-culture of Saccharomyces cerevisiae and Williopsis saturnus. S. Afr. J. Enol. Vitic. 31, 116-124.

Verwaal, R., Jiang, Y., Wang, J., Daran, J.-M., Sandmann, G., Van den Berg, J.A. \& Van Ooyen, A.J., 2010. Heterologous carotenoid production in Saccharomyces cerevisiae induces the pleiotropic drug resistance stress response. Yeast 27, 983-998.

Vilanova, M., Ugliano, M., Varela, C., Siebert, T., Pretorius, I.S. \& Henschke, P.A., 2007. Assimilable nitrogen utilisation and production of volatile and non-volatile compounds in chemically defined medium by Saccharomyces cerevisiae wine yeasts. Appl. Microbiol. Biotechnol. 77, 145-157.

Wang, Y.-Q., Ye, D.-Q., Zhu, B.-Q., Wu, G.-F. \& Duan, C.-Q., 2014 Rapid HPLC analysis of amino acids and biogenic amines in wines during fermentation and evaluation of matrix effect. Food Chem. 163, 6-15.

Zhang, M., Pan, Q., Yan, G. \& Duan, C., 2011. Using headspace solid phase micro-extraction for analysis of aromatic compounds during alcoholic fermentation of red wine. Food Chem. 125, 743-749. 